\title{
LAS PRIORIDADES DE UN LÍDER COMO JESÚS |
}

Azo Salazar, Segundo Universidad Peruana Unión sazodoc@gmail.com

Jesús desafió a sus discípulos para elegir entre el modelo de liderazgo según los gobernantes de este mundo y el modelo de liderazgo ejemplificado a través de una vida de servicio abnegado en favor de los demás. Frente a ello, los líderes de hoy deberian hacerse las preguntas: ¿Qué modelo de liderazgo estoy siguiendo hoy? ¿Estoy buscando la fama y la popularidad al tratar de desarrollar "lo bueno" que hay en el ser humano? Pero la pregunta más importante es acerca de las prioridades de Jesús como líder. Él le dio el primer lugar a su Padre; el segundo lugar a su familia y, en tercer lugar, a la misión de hacer discípulos. Finalmente, el autor desafía al lector a liderar como Jesús, ordenando sus prioridades en su agenda diaria, en base al modelo dejado por el Señor Jesús.

Palabras clave: Misión, discipulado, liderazgo, prioridades. 
Summary $\quad$ Jesus challenged his disciples to choose between the leadership models of the rulers of this world, or imitate the model of leadership exemplified by a life of unselfish service for others. Thus the leaders of today should ask themselves the following question: What leadership model do I follow today? Am I looking for fame and popularity while trying to develop "the good" there is in a person?. But the most important question is about the priorities of Jesus as a leader. He gave his Father first place, while placing his family as second, and then finally in third place was his mission to make disciples. Finally, the author challenges the reader to lead like Jesus, ordering their priorities in their daily schedule as the model Jesus left for us.

Keywords: Mission, discipleship, leadership, priorities. 


\section{Introducción}

El diccionario de la lengua española define el término prioridad como anterioridad de una cosa respecto de otra, en tiempo o en orden. Mientras que prioritario dícese de lo que tiene prioridad respecto de algo. ${ }^{1}$ Tomando en cuenta estos conceptos de prioritario, uno se pregunta:

¿Cuáles fueron las prioridades que Jesús tuvo como líder? ¿Cuál fue la mayor lucha que Jesús tuvo que sostener? Como ser humano que era ¿cómo ordenó 166 su agenda de actividades frente a los múltiples compromisos que tenía cada día? Por cierto, no fue fácil para Jesús escapar a los compromisos, cuando su fama llegó a ser conocida y las multitudes lo buscaban desde la primera hora de cada mañana.

Si Jesús hubiese nacido en el siglo XXI ¿cuáles hubiesen sido sus prioridades como líder en una sociedad posmoderna que, ante la demanda de lo que es prioritario, siempre contesta "no hay tiempo", "todo es rela-

1 Diccionario de la Real Academia Española (Editorial Espasa Calpe: Madrid 1984), 1105. tivo" o "nada es absoluto"?

Existen tres preguntas que se proponen frente a esta temática y son: ¿Qué modelo de liderazgo estoy siguiendo? ¿Cuáles eran las prioridades del liderazgo de Jesús? y ¿cómo puedo liderar como lideró Jesús?

\section{¿Qué modelo de liderazgo estoy siguiendo?}

La respuesta a esta pregunta fue respondida por Jesús a sus discípulos, en ocasión del pedido que le hicieran Jacobo y Juan respecto a ocupar una posición de honor en su gloria. "Mas Jesús, llamándoles, les dijo: Sabéis que los que son tenidos por gobernantes de las naciones se enseñorean de ellas, y sus grandes ejercen sobre ellas potestad.

Pero no será así entre vosotros, sino que el que quiera hacerse grande entre vosotros será vuestro servidor, y el que de vosotros quiera ser el primero, será siervo de todos. Porque el Hijo del Hombre no vino para ser servido, sino para servir, y para dar su vida en rescate por muchos". (Mr 10:42-45) 
White declara que el pedido de Jacobo y Juan, a través de su madre, había dejado entrever su egoísmo y su deseo de buscar preferencia sobre sus hermanos $^{2}$, mientras que el modelo que Jesús les estaba mostrando, a través de su propia vida, era un liderazgo de servicio en favor de los demás.

Jesús había confrontado a sus discípulos el modelo de liderazgo según el mundo secular, donde los que asumen el liderazgo de las naciones, se enseñorean de ellas, con el nuevo modelo de liderazgo según Jesús, caracterizado por una vida altruista en favor de los demás.

El mundo del conocimiento está lleno de libros de liderazgo, desde Ted Engstron, Bill Hybels, Crawford Loritts, hasta John Maxwel, Rick Warren y John Stott, la mayoría de ellos con muy buenas ideas centradas en desarrollar un liderazgo cristiano. Sin embargo, son pocos los escritores que se han ocupado de escribir acerca del modelo de Jesús como

2 Elena G. de White, El Deseado de todas las gentes (Mountain View, California: Publicaciones Interamericanas, 19955), 502. líder y, específicamente, acerca de sus prioridades como líder.

Es fácil perderse al tratar de imitar a líderes de opinión o lideres juveniles, donde el ser humano es el centro de atención de cada vez más seguidores. Estos miden su popularidad cada día a través de las redes de comunicación como Facebook, Twitter, Youtube, etc.

La mayoría de estos líderes a los cuales el mundo sigue, a través de las redes sociales, han sido influenciados por el pensamiento posmoderno que con sus actitudes declaran que Dios no puede ser el centro de la vida, que todo es relativo en cuanto a valores y que no existen absolutos.

Frente a ello, es necesario responder a la siguiente pregunta:

\section{¿Estoy siguiendo el modelo de liderazgo que Jesús dejó a sus seguidores?}

En esta sección nos proponemos analizar la declaración bíblica de Lucas 5:15-16: "Pero su fama se extendía más y más; y se reunía mucha gente para oírle, y 
para que les sanase de sus enfermedades. Más él se apartaba a lugares desiertos, y oraba”.

El contexto inmediato de la declaración lucana nos muestra que Jesús había sanado a un leproso (vv.12-14), lo que hizo que la fama de Jesús se extendiera más y más, y su tiempo podía ser absorbido por el hacer antes que por el ser.

"La fama de Jesús se extendía más y más", indica como las multitudes se reunían alrededor de Jesús para oírle "y para que les sa168 nase de sus enfermedades", aun cuando Él había pedido al leproso que había sido sanado que no lo dijera a nadie $($ v. 14,15$)$.

White declara: "Pero su divulgación del asunto estorbó la obra de Salvador. Hizo que la gente acudiese a Él en tan densas muchedumbres que, por un tiempo, se vio obligado a suspender sus labores".

La declaración lucana "la fama de Jesús se extendía más y más", indica también, de ma-

3 White, El Deseado de todas las gentes, 230. nera objetiva y real, que la cantidad de seguidores de Jesús se estaba propagando rápidamente a través de los lugares donde él pasaba.

Por cierto que no era este el propósito que Jesús tenía al sanar a través de milagros, puesto que solo "lo hacía para bendición de la humanidad y para revelar el carácter de Dios" ${ }^{4}$ frente al dolor humano.

Hoy en día en las redes sociales la persona más famosa es la que tiene mayor número de seguidores. Así la persona con más seguidores en el mundo es Lady Gaga. La excéntrica cantante tiene, nada más ni nada menos, casi 26 millones de seguidores. En segundo puesto aparece el ídolo y cantante juvenil canadiense Justin Bieber, con 23.5 millones de seguidores. La cantante Katy Perry reúne casi 22 millones. La cantante Rihanna, que ocupa el cuarto puesto, llega a 21 millones y, en el quinto puesto, la cantante Britney Spears que aparece con unos 18 millones de seguidores.

4 Ibid., 373. 
Uno de los pocos famosos, que no es cantante, figura en el sexto lugar: Barack Obama, el presidente de Estados Unidos, que ostenta 17 millones de seguidores. Le sigue Shakira, la única latina de la lista, con 16 millones. Taylor Swift, la cantante estadounidense de música country, suma 15 millones de seguidores en el Twitter. En noveno lugar está la modelo Kim Kardashian, que también suma 15 millones de seguidores. Por último, la exitosa cantante Nicki Minaj cierra el ranking con 13 milloncitos de seguidores. ${ }^{5}$

La fama y la popularidad terminaron por convertirse en una poderosa tentación en la vida de Jesús como líder, tentación que podría haber cambiado sus prioridades.

Lucas continúa diciendo que "se reunía a mucha gente para oírle, y para que les sanase de sus enfermedades". Jesús no rehuyó su responsabilidad de enseñar

5 Ciudad.com, "La lista de los 10 famosos del mundo más seguidos en Twitter", http:// www.ciudad.com.ar/espectaculos/94687/ lista-10-famosos-mundo-mas-seguidos-twitter (consultado: 27 de abril, 2013). y sanar a aquellos que venían a Él. En realidad esa había sido la misión que anunció cumpliría, la de "dar buenas nuevas a los pobres;...sanar a los quebrantados..." (Lc 4:18).

El problema estuvo en que las multitudes, así como sus discípulos, entendieron mal el propósito y siguieron a Jesús para poder "hacerle rey" (Jn 6:15).

\section{La principal prioridad de Jesús como líder: el Padre}

Sin embargo, Jesús tenia bien definida su visión (Lc 19:10) puesto que su principal prioridad, como líder, fue depender enteramente de su Padre y no hacer nada que pudiera separarle de Él.

El evangelio de Lucas declara cuál fue la actitud de Jesús frente a sus seguidores y a la fama cada vez más creciente al decir: "Pero Él se apartaba a lugares desiertos y oraba” (Lc 5:16).

La conjunción pero, en el idioma original es adversativa e indica que lo que Jesús hizo a continuación es contrario u opuesto 
a lo que venía haciendo. ${ }^{6}$ Probablemente la gente cuestionó el hábito de Jesús de aislarse de las multitudes para encontrar tiempo para orar, lo que podría ser considerado algo extraño frente a los requerimientos de las multitudes, especialmente de los enfermos.

Jay Dennis escribiendo acerca de el hábito de Jesús de aislarse declara que:

El hábito de Jesús de aislarse, momentáneamente o por un largo tiempo, tenía como propósito retirarse del ruido y las demandas cotidianas para pasar tiempo con Dios, relacionarse con Él y permitir que se recargaran sus baterias emocionales y espirituales. Este hábito provee el momento oportuno para elaborar su programa de actividades diarias, no importa cuán ocupado y complicado pueda ser ese programa. Sin embargo, en nuestra sociedad, encontrar un lugar para recluirse se hace cada vez más difícil.?

6 Roberto Hanna, Sintaxis exégetica del Nuevo Testamento griego (El Paso, Texas: Editorial Mundo Hispano, 1997), 63.

7 Dennis, Jay, Los hábitos de Jesús: prac-
Por tanto, la prioridad de Jesús, como líder, fue hecha principalmente en función de su relación de dependencia que mantuvo con su Padre. Su santo y seña era "No puedo yo hacer nada por mí mismo...porque no busco mi voluntad, sino la voluntad del que me envió, la del Padre". (Jn 5:30).

La mayor tentación que Jesús enfrentó cada día fue, precisamente, la de dejar de depender del poder de su Padre, para depender de sí mismo y en esto radicó la primera tentación que Satanás le infligió en el desierto, cuando le pidió: "Si eres Hijo de Dios, di que estas piedras se conviertan en pan" (Mt 4:3).

Tan grande era su relación de dependencia del Padre que pudo decir: "...Las palabras que yo os hablo, no las hablo por mi propia cuenta, sino que el $\mathrm{Pa}$ dre que mora en mí, El hace las obras" (Jn 14:10).

tiquemos la disciplina del Maestro (El Paso, Texas: Editorial Mundo Hispano, 2005). Ken Blanchard y Phil Hodges. Un lider como Jesús (Nashville: Editorial Grupo Nelson, 2006), 11-22. 
Se puede afirmar sin temor a dudas que, en la agenda de Jesús, la comunión personal con su Padre ocupaba el primer lugar, el mejor y el más importante e impostergable.

\section{La segunda prioridad de Jesús como líder: su familia}

Habiendo puesto en práctica Jesús, en su vida, el primer gran mandamiento: "Amarás al Señor tu Dios con todo tu corazón, y con toda tu alma, y con toda tu mente" (Mt 22:37). La segunda prioridad en su vida como líder estuvo en relación con el segundo gran mandamiento: "Amarás a tu prójimo como a ti mismo" (v. 39). En este sentido, su prójimo inmediato fue su propia familia (María, José y sus hermanos).

El evangelio de Lucas registra que Jesús, después del reencuentro con sus padres en Jerusalén, "descendió con ellos y volvió a Nazaret y estaba sujeto a ellos" (Lc 2:51). En realidad sin descuidar los negocios de su Padre (Dios), Jesús acompañó y proveyó para las necesidades de su familia durante los primeros treinta años, antes de dar inicio a su ministerio público (Lc 3:23).

Durante los tres años y medio que duró su ministerio, Jesús regreso a su hogar en $\mathrm{Na}$ zaret (Mt 13:54), porque consideraba que había una obra que hacer especialmente entre sus hermanos incrédulos (Jn 7:1-5), y también porque era su deber honrar a su madre, la cual probablemente era ya una viuda.

Bastante conocida fue la actitud de respeto y amor que Jesús manifestó hacia su madre en los últimos instantes de su vida, pues no se olvidó que estaba en su agenda el hacer previsión para su cuidado, de allí que olvidándose de sí mismo le dijo a Juan, su discípulo: "He ahí tu madre. Y desde aquella hora el discípulo la recibió en su casa" (Jn 19:26-27).

Los resultados de haberle dedicado a su familia un lugar de prioridad, después de su $\mathrm{Pa}$ dre, influyeron poderosamente en la conversión de sus hermanos incrédulos, a quienes Lucas menciona entre el grupo de 
aquellos que estaban en el aposento alto previo al Pentecostés (Hch 1:14).

La tercera prioridad de Jesús como líder: sus discípulos

Después de su comunión personal que Jesús mantuvo siempre con su Padre, y del tiempo que dedico para estar sujeto a su familia, la siguiente prioridad en la agenda de Jesús como líder consistió en llamar a doce seguidores (Mt 10:1-4), y después a otros setenta (Lc 10:1), a quienes

172 les dedicó el tiempo necesario para enseñarles, por precepto y por el ejemplo, cómo llegar a ser verdaderos discípulos en el reino de Dios.

A este grupo selecto Jesús dedicó tres años y medio, pues ellos serían el núcleo que daría origen a la naciente Iglesia cristiana. Caminó, comió y durmió junto con ellos. Les enseñó los principios del reino (Mt 5:1-7:29), así como los principios del trabajo en equipo (Mt 10:5-15; 11:1; Lc 10:1-12).

Aun cuando Jesús sabía que serían tardos para entender todo lo que él les enseñaba (Lc 24:25), sembró en sus corazones la semilla del evangelio, y la regó cada día con oración, amor, paciencia y tolerancia. El fruto del Espíritu (Gal 5:22-23) se dejó ver luego de su ascensión en el día del Pentecostés (Hch 2), cuando Pedro, en su primer sermón, vio unirse a la Iglesia a tres mil (Hch2:41), luego a cinco mil (Hch 4:4) y luego "aumentaban más, gran número, asi de hombres como de mujeres" (Hch 5:14).

Jesús al llamar a este pequeño grupo, lo hizo después de haber dedicado toda la noche a orar por ellos (Lc 6:12-16), les enseñó a orar (Lc 11:1-4) y, antes de finalizar su ministerio, oró al Padre por cada uno de ellos (Jn 17:1-12), con la esperanza de que, cumplido su ministerio que les fue encomendado, un día pudieran estar con Él en su segunda venida (Jn 17:24).

\section{¿Cómo puedo liderar como lideró Jesús?}

Teniendo en cuenta que "el líder no nace, ni se hace, sino 
que Dios lo hace”, cada líder que quiere liderar como lideró Jesús, debe seguir su ejemplo. En primer lugar, debe vivir una comunión personal íntima con Dios, para luego poder enseñar a sus seguidores por precepto y ejemplo a hacer de ello su primer trabajo:

Jesús les mostró cómo orar Él oró muy temprano por la mañana (Mc 1:35) preparándose para la batalla espiritual del día, y hubo ocasiones en que oró toda la noche (Lc $6: 12 ; 11: 1-4)$.

Cómo oraba Jesús. Él oró con las personas y no solamente por ellas (Lc 9:28). Por tanto, tome tiempo para orar con su familia y amigos. Si usted promete orar, ore inmediatamente o escriba su propósito para orar después.

Él oró continuamente. Jesús nunca dejó de orar. "La oración fue para Él una necesidad y un privilegio". ${ }^{8}$ Les

8 Elena G. de White, El camino a Cristo (Buenos Aires, Argentina: Asociación Casa Editora Sudamericana, 1976), 93. refirió también, a sus seguidores, una parábola acerca de la necesidad de orar siempre y no desmayar (Lc 18:1).

Jesús oró para glorificar a su Padre, al declarar. "Estas cosas habló Jesús, y levantando los ojos al cielo, dijo: Padre, la hora ha llegado; glorifica a tu Hijo, para que también tu Hijo te glorifique a ti” (Jn 17:1).

Antes de tomar grandes decisiones. "En aquellos días Él fue al monte a orar, y pasó la noche orando a Dios. Y cuando era de día, llamó a sus discípulos, y escogió a doce de ellos, a los cuales también llamó apóstoles" (Lc 6:12-13).

Para vencer la tentación. Y saliendo, se fue, como solía, al monte de los Olivos; y sus discípulos también le siguieron. Cuando llegó a aquel lugar, les dijo: "Orad para que no entréis en tentación. Y Él se apartó de ellos a distancia como de un tiro de piedra; y puesto de rodillas oró" (Lc 22:39-41). 
Para interpretar correctamente la Palabra de Dios. "Y vino a Él el tentador, y le dijo: Si eres Hijo de Dios, di que estas piedras se conviertan en pan. Él respondió y dijo: Escrito está: No solo de pan vivirá el hombre, sino de toda palabra que sale de la boca de Dios" (Mt 4:3, 4).

Para no perder de vista el propósito de su misión. Levantándose muy de mañana, siendo aún muy oscuro, salió y se fue a un lugar desierto, y allí oraba. Y le buscó Simón, y los que con él estaban; y hallándole, le dijeron: Todos te buscan. Él les dijo: Vamos a los lugares vecinos, para que predique también allí; porque para esto he venido (Mr 1:35-38).

Jesús tenía bien claras sus prioridades de liderazgo, así como el propósito por el cual había venido: el de anunciar las buenas nuevas de salvación a todos no apenas a un grupo que trataba de detenerle para que se quedara con ellos.
Los líderes de hoy son tentados a perder de vista sus prioridades, cuando dejan de depender de Dios y pierden de vista a Jesús como modelo de liderazgo y lo más trágico es que pierden el propósito principal del "Id y haced discípulos".

Este es el modelo de liderazgo que Jesús dejó a sus seguidores, al mostrar que un líder debe ser, principalmente, un hombre o mujer de oración, espiritual, que en sus palabras y hechos demuestre que depende enteramente de Dios el Padre, como lo hizo Jesús. Blanchard declara que:

Las razones espirituales para guiar como Jesús son tan apremiantes como desafiantes en un mundo donde los esfuerzos egocéntricos para la autogratificación, la autopromoción y la autoprotección describen el tipo de liderazgo en todo nivel de las relaciones humanas. ${ }^{9}$

Cuando el líder, en su afán de buscar protagonismo y popularidad o sencillamente por querer conseguir resultados, deja de

9 Blanchard, Un lider como Jesús, 185. 
depender del Padre para depender de sus esfuerzos o capacidades, entonces pierde de vista sus prioridades, y se hace daño a sí mismo y a sus liderados.

La mayor tentación de Jesús fue actuar independientemente de su Padre, sin la ayuda y dirección del Espíritu Santo. Por ello, comprendiendo la mayor lucha del líder consigo mismo, dijo a sus discípulos: "el Espíritu está dispuesto pero la carne es débil" (Mr 14:38).

Esta es la mayor lucha que cada líder tiene que sostener cada mañana y cada instante del día. Esta es la batalla de la fe que cada joven debe pelear cada día, con la seguridad de que la victoria le es asegurada si cultiva el hábito de aprender a depender enteramente del Padre, como Jesús lo hizo.

\section{Conclusiones}

Los modelos de liderazgo, centrados en desarrollar apenas "lo bueno" que hay en el interior del ser humano, desconocen la cosmovisión bíblica de la separación del hombre de Dios por causa del pecado, y terminan por exaltar el egoísmo humano.

1. Las prioridades de Jesús, como líder, fueron hechas principalmente en función de la relación de dependencia que mantuvo con su Padre a través de su comunión personal las veinticuatro horas del día.

2. La victoria de los líderes de hoy estará asegurada en la medida que mantengan sus prioridades de liderazgo en entera dependencia del $\mathrm{Pa}$ dre, a través de una vida de comunión permanente.

3. Cuando los líderes de hoy solo procuran la popularidad y la fama, a través de la búsqueda de resultados, pierden de vista el modelo de liderazgo de Jesús y por ende sus prioridades, para terminar dependiendo de si mismos.

4. Los líderes de hoy deberían imitar el modelo de liderazgo de Jesús, al ordenar su agenda diaria de prioridades: Dios, la familia y el hacer discípulos. 